\title{
Integral Partitioning Approach to Stability Analysis and Stabilization of Distributed Time Delay Systems *
}

\author{
Zhiguang Feng* and James Lam* \\ * Department of Mechanical Engineering, University of Hong Kong, \\ Pokfulam Road, Hong Kong, P. R. China \\ (e-mail: congdian@gmail.com; e-mail: james.lam@hku.hk)
}

\begin{abstract}
In this paper, the problems of delay-dependent stability analysis and stabilization are investigated for linear continuous-time systems with distributed delay. By introducing an integral partitioning technique, a new form of Lyapunov-Krasovskii functional (LKF) is constructed and improved distributed delay dependent stability conditions are established in terms of linear matrix inequalities (LMIs). Based on the criteria, a design algorithm for a state feedback controller is proposed. The results developed in this paper are less conservative than existing ones in the literature, which is illustrated by several examples.
\end{abstract}

Keywords: Distributed Delay; Delay Dependent; Integral Partitioning; Linear Matrix Inequalities; Stabilization.

\section{INTRODUCTION}

Time delay, often encountered in modern complex engineering systems involving multitude of information and communication networks, is the main causes of instability and poor performance of dynamic systems Dugard and Verriest [1998], Li and de Souza [1997]. Therefore, timedelay systems have attracted many researchers' attention and have been extensively studied in the literature, see Gao et al. [2008], Wang et al. [2008] and Xu and Lam [2008]. Stability criteria for time-delay systems can be classified into two categories: delay-independent and delay-dependent criteria. Generally, the conservatism of delay-dependent stability conditions is less than that of delay-independent ones especially when the delay is small. Recently, the development of the techniques for delaydependent stability analysis has been focusing on the effectiveness in reducing conservatism of stability conditions. To mention a few, for linear systems with constant timedelay, a delay-dependent stability criterion is proposed based on some model transformation techniques in Moon et al. [2001]. By introducing the slack variables to deal with the weighted cross products of the state and the delayed state, the new stability criterion obtained in $\mathrm{Xu}$ and Lam [2005] is less conservative than the result in Moon et al. [2001]. Gouaisbaut and Peaucelle [2006] employ the delay-partitioning approach to further reduce the conservatism of the result in Xu and Lam [2005]. Benefiting from the delay-partitioning approach, many results for other systems are extended. To mention a few, the stability of recurrent neural networks with time-invariant delay is studied in Du and Lam [2009]; the stabilization results for discrete singular delay systems are given in Feng et al. [Published online: 20 DEC 2010.]; the stabilization result of Markovian jump systems with time delay is presented in

\footnotetext{
^ This work was partially supported by GRF HKU 7137/09E.
}

Fei et al. [2009]. For systems with time-varying delay, the stability problem is investigated by using the free weight matrices method in $\mathrm{He}$ et al. [2007]. Making use of the delay bound information, a new Lyapunov functional is presented in Jiang and Han [2008] which improved the result in He et al. [2007]. With a new method to estimate the time derivative of the Lyapunov functional, less conservatism results are obtained in Shao [2009]. Recently, by using the delay partitioning-based Lyapunov Functionals, better results are given in Fridman et al. [2009].

It is noted that the results mentioned above are derived for systems with discrete delay. Another type of timedelay, namely, distributed delay, will appear when the number of summands in a system equation is increased and the differences between neighboring argument values are decreased $\mathrm{Xu}$ and Chen [2004]. Systems with distributed delay can be applied in the modeling of feeding systems and combustion chambers in a liquid monopropellant rocket motor with pressure feeding Fiagbedzi and Pearson [1987]. Therefore, a growing attention has been devoted to studying distributed delay systems in recent years, see Li et al. [2009], Li et al. [2008] and Yue and Han [2005]. For $H_{\infty}$ control problem, taken two types of linear systems with distributed time delay into account, $H_{\infty}$ controllers are designed in Xie et al. [2001]. Different from the method used in Xie et al. [2001], the descriptor discretized Lyapunov-Krasovskii Functionals (LKFs) is constructed to derive an improved results on $H_{\infty}$ control problem in Fridman [2009]. Based on the parameter dependent Lyapunov functional approach, the robust $H_{\infty}$ control problem for a class of uncertain distributed delay systems subject to real convex polytopic-type uncertainties is investigated in $\mathrm{Wu}$ et al. [2007]. For the problem of filtering, Xu et al. [2005] design robust $H_{\infty}$ filter for linear uncertain systems with both discrete and distributed delays, while the problem is investigated for descriptor systems in Yue and Han 
[2004]. However, the discrete delay and distributed delay are assumed to be known real constants in Xu et al. [2005 and Yue and Han [2004]. Wu et al. [2006] study robust $H_{\infty}$ and $L_{2}-L_{\infty}$ filtering for the LPV systems with timevarying discrete and distributed delays. For stability analysis and stabilization results, robust stabilization conditions in terms of linear matrix inequalities (LMIs) are presented in Zheng and Frank [2002], and the obtained results are applied to stabilize the combustion in rocket motor chambers. Gu [2003] uses Jensen's inequality and variable elimination method of matrix inequality to improve the stability criterion of systems with distributed delay. However this method is complicated and is difficult to extend to synthesis problems. By using the analytic solution to Lyapunov functional equations of distributed delay systems, a necessary and sufficient condition for distributed delay systems with unknown but bounded constant delay is proposed in Suh et al. [2006]. Chen and Zheng [2007] use the descriptor system approach provided in Fridman [2001] to investigate the stability of neutral systems with discrete and distributed delays. Because the stability conditions in Chen and Zheng [2007] is neutral-delay-independent, the conservatism is larger than the neutral-delay-dependent results obtained in $\mathrm{Li}$ and $\mathrm{Zhu}$ [2008]. By introducing some triple integral terms into LKFs, the conservatism of the results in Li and Zhu [2008] is further reduced in Sun et al. [2009]. It should be pointed out that conservatism is still serious in these results, which is the motivation for this study.

In this paper we aim to further reduce the conservatism of existing results for stability criteria of linear distributed delay systems. An improved version of distributed delay dependent condition in terms of LMIs is established by employing the integral partitioning technique. Based on this, a delay-dependent sufficient condition for the existence of a state feedback controller which guarantees stability of the closed-loop system. In addition to delay dependence, the obtained results are also dependent on the partitioning size. The conservatism will decrease with the increase of partitioning size. Finally, numerical examples are given to illustrate the effectiveness of the presented results.

The rest of this paper is briefly outlined as follows. In Section 2, the problem of stabilization is formulated and some definitions and lemmas are given. The stability analysis results for distributed systems and the corresponding design method of state-feedback controller are presented in Section 3. Illustrative examples are provided in Section 4 to show the reduced conservatism of our results. We conclude the paper in Section 5 .

Notation: The notation used throughout the paper is standard. $\mathbb{R}^{n}$ denotes the $n$-dimensional Euclidean space and $P>0(\geq 0)$ means that $P$ is real symmetric and positive definite (semi-definite); $I$ and 0 refer to the identity matrix and zero matrix with compatible dimensions; $A^{T}$ and $A^{-1}$ denote the transpose and the inverse of a matrix $A$; $\star$ stands for the symmetric terms in a symmetric matrix and $\operatorname{sym}(A)$ is defined as $A+$ $A^{T}$. Matrices are assumed to be compatible for algebraic operations if their dimensions are not explicitly stated.

\section{PROBLEM FORMULATION}

Consider a class of linear continuous-time distributed time delay systems described by

$$
\left\{\begin{array}{l}
\dot{x}(t)=A x(t)+A_{h} \int_{t-h}^{t} x(s) d s+B u(t) \\
x(t)=\varphi(t), \forall t \in[-2 h, 0]
\end{array}\right.
$$

where $x(t) \in \mathbb{R}^{n}$ is the state vector; $u(t) \in \mathbb{R}^{q}$ is the vector of control input; $h>0$ represents the system distributed delay; $A, A_{h}$ and $B$ denote constant matrices with appropriate dimensions; $\varphi(t)$ is the initial function. Because the term $\int_{t-\frac{(m+1) h}{m}}^{t-h} x(s) d s$ ( $m$ is a positive integer) will be used in the proof of Theorem 1 , the initial condition is given on $[-2 h, 0]$.

The goal of the paper is to derive less conservative delaydependent stability criteria to ensure a larger maximum upper bound on the distributed delay. Based on this, we will design a static state feedback controller

$$
u(t)=K x(t)
$$

such that the closed-loop system

$$
\dot{x}(t)=(A+B K) x(t)+A_{h} \int_{t-h}^{t} x(s) d s
$$

is stable for a given $h$.

In order to analyze the stability of system (1), the following integral inequalities are required.

Lemma 1. Sun et al. [2009] For any matrix $M>0$ and a scalar $\gamma>0$, if there exists a Lebesque vector function $w:[0, \gamma] \rightarrow \mathbb{R}^{n}$, then the following inequalities hold:

$$
\begin{gathered}
-\int_{t-\gamma}^{t} w^{T}(s) M w(s) d s \\
\leq-\frac{1}{\gamma}\left(\int_{t-\gamma}^{t} w^{T}(s) d s\right) M\left(\int_{t-\gamma}^{t} w(s) d s\right) \\
\leq-\frac{2}{\gamma^{2}}\left(\int_{-\gamma}^{0} \int_{t+\theta}^{t} w^{T}(s) d s d \theta\right) M\left(\int_{-\gamma+\theta}^{0} \int_{t+\theta}^{t} w(s) d s d \theta\right)
\end{gathered}
$$

Proof. The inequality in (4) has essentially been proved in $\mathrm{Gu}$ [2000]. For inequality (5), the proof can be carried out following the similar line as in the proof of Lemma 1 in $\mathrm{Gu}$ [2000]. Using Schur complement, it is easy to see that

$$
\left[\begin{array}{cc}
w^{T}(s) M w(s) & w^{T}(s) \\
\star & M^{-1}
\end{array}\right] \geq 0
$$

for any $t-\gamma \leq s \leq t$. Integrating the above inequality over the triangle defined by $t-\gamma \leq s \leq t$ and $-\gamma \leq \theta \leq 0$ yields 


$$
\left[\int_{-\gamma}^{0} \int_{t+\theta}^{t} w^{T}(s) M w(s) d s d \theta \int_{-\gamma}^{0} \int_{t+\theta}^{t} w^{T}(s) d s d \theta\right] \geq 0
$$

Using the Schur complement again, we have that (5) holds.

\section{MAIN RESULTS}

In this section, the improved sufficient condition is derived firstly by employing the integral partitioning technique, which guarantees that the system in (1) is stable. The result of stability analysis for system (1) is presented in the following theorem.

Theorem 2. For a given scalar $h$, the system in (1) is asymptotically stable, if there exist matrices $P>0, Q>0$, $Z>0$ and $R>0$ such that the following LMI holds:

$$
\Omega<0
$$

where

$$
\begin{aligned}
\Omega & =\operatorname{sym}\left(W_{P}^{T} P W_{S}\right)+W_{Q}^{T} \bar{Q} W_{Q}+W_{Z}^{T} \bar{Z} W_{Z}+W_{R}^{T} \bar{R} W_{R} \\
W_{P} & =\left[\begin{array}{ll}
I_{n} & 0_{n,(m+1) n}
\end{array}\right], W_{S}=\left[\begin{array}{lll}
A & A_{h} \cdots A_{h} & 0_{n, n}
\end{array}\right] \\
W_{Z} & =\left[\frac{I_{n} 0_{n,(m+1) n}}{0_{n, n} I_{n} 0_{n, m n}}\right] \\
W_{R} & =\left[\frac{A A_{h} \cdots A_{h} 0_{n, n}}{\frac{h}{m} I_{n}-I_{n} 0_{n, m n}}\right] \\
W_{Q} & =\left[\begin{array}{ccc}
\frac{0_{m n, n} I_{m n} 0_{m n, n}}{0_{m n, 2 n} I_{m n}}
\end{array}\right] \\
\bar{R} & =\left[\begin{array}{cc}
\frac{1}{2}\left(\frac{h}{m}\right)^{2} R & 0_{n, n} \\
\star & -2\left(\frac{m}{h}\right)^{2} R
\end{array}\right] \\
\bar{Q} & =\left[\begin{array}{ccc}
Q & 0_{m n, m n} \\
\star & -Q
\end{array}\right], \bar{Z}=\left[\begin{array}{cc}
\frac{h}{m} Z & 0_{n, n} \\
\star & -\frac{m}{h} Z
\end{array}\right]
\end{aligned}
$$

Proof. Construct a new Lyapunov functional candidate as

$$
\begin{aligned}
V(x(t)) & =x^{T}(t) P x(t)+\int_{-\frac{h}{m}}^{0} \int_{\theta}^{0} \int_{t+\lambda}^{t} \dot{x}^{T}(s) R \dot{x}(s) d s d \lambda d \theta \\
& +\int_{t-\frac{h}{m}}^{t} \eta^{T}(\theta) Q \eta(\theta) d \theta+\int_{-\frac{h}{m}}^{0} \int_{t+\theta}^{t} x^{T}(s) Z x(s) d s d \theta
\end{aligned}
$$

where

$$
\eta(t)=\left[\begin{array}{c}
\int_{t-\frac{h}{m}}^{t} x(s) d s \\
\vdots \\
t-\frac{(m-2) h}{m} \\
\int_{t-\frac{(m-1) h}{m}}^{m} x(s) d s \\
\int_{t-h}^{m} x(s) d s
\end{array}\right] \in \mathbb{R}^{m n}
$$

Evaluating the derivative of $V(x(t))$ along the solutions of system (1), we obtain

$$
\begin{aligned}
\dot{V}(x(t)) & =2 x^{T}(t) P \dot{x}(t) \\
& +\eta^{T}(t) Q \eta(t)-\eta^{T}\left(t-\frac{h}{m}\right) Q \eta\left(t-\frac{h}{m}\right) \\
& +\frac{h}{m} x^{T}(t) Z x(t)-\int_{t-\frac{h}{m}}^{t} x^{T}(s) Z x(s) d s \\
& +\frac{1}{2}\left(\frac{h}{m}\right)^{2} \dot{x}^{T}(t) R \dot{x}(t) \\
& -\int_{-\frac{h}{m}}^{0} \int_{t+\theta}^{t} \dot{x}^{T}(s) R \dot{x}(s) d s d \theta
\end{aligned}
$$

By Lemma 1, we have

$$
\begin{gathered}
-\int_{t-\frac{h}{m}}^{t} x^{T}(s) Z x(s) d s \leq-\frac{m}{h} \int_{t-\frac{h}{m}}^{t} x^{T}(s) d s Z \int_{t-\frac{h}{m}}^{t} x(s) d s \\
-\int_{-\frac{h}{m} \int_{t+\theta}^{t} \dot{x}^{T}(s) R \dot{x}(s) d s d \theta}^{t} \\
\leq-2\left(\frac{m}{h}\right)^{2} \int_{-\frac{h}{m}}^{0} \int_{t+\theta}^{t} \dot{x}^{T}(s) d s d \theta R \int_{-\frac{h}{m}}^{0} \int_{t+\theta}^{t} \dot{x}(s) d s d \theta
\end{gathered}
$$

Substituting (9)-(10) into (8) yields

$$
\dot{V}(x(t)) \leq \zeta^{T}(t) \Omega \zeta(t)
$$

where

$$
\zeta(t)=\left[\begin{array}{c}
x(t) \\
\eta(t) \\
\int_{t-\frac{(m+1) h}{m}}^{t-h} x(s) d s
\end{array}\right] \in \mathbb{R}^{(m+2) n}
$$

Therefore, if $\Omega<0, \dot{V}(x(t))<0$ is derived and system (1) is asymptotically stable. This completes the proof.

Remark 3. The main technique utilized in this paper is the integral partitioning idea which partitions the integral interval into $m$ equal subintervals. When the delay interval $[-h, 0]$ is nonuniformly divided into $\mathrm{m}$ segment, i.e., 
$[-h, 0]=\bigcup_{i=1}^{i=m} h_{i}$ with $0=h_{0}<h_{1}<\cdots<h_{m}=h$, we can construct a new Lyapunov functional candidate as

$$
\begin{aligned}
\check{V}(x(t)) & =x^{T}(t) P x(t)+\sum_{i=1}^{m} \int_{-h_{i}}^{-h_{i-1}} \int_{\theta}^{0} \int_{t+\lambda}^{t} \dot{x}^{T}(s) R \dot{x}(s) d s d \lambda \\
& +\sum_{i=1}^{m} \int_{-h_{i}}^{-h_{i-1}} \int_{t+\theta}^{t} x^{T}(s) Z x(s) d s d \theta d \theta
\end{aligned}
$$

Remark 4. For systems with constant discrete delay, Gouaisbaut and Peaucelle present the delay partitioning method to significantly improve the stability analysis criterion in Gouaisbaut and Peaucelle [2006]. Motivated by this technique, we first investigate the integral partitioning method to partition the integral for systems with distributed delay in order to further reduce the conservatism of existing stability results.

Next, we will present the controller design method for the system in (1) based on Theorem 1 such that the closedloop system is stable.

Theorem 5. For given scalar $h$, the system in (1) is asymptotically stable, if there exist matrices $\tilde{P}>0, \tilde{Q}>0$, $\tilde{Z}>0, \tilde{R}>0$ and matrix $L$ such that the following LMI hold:

$$
\left[\begin{array}{cc}
\tilde{\Omega} & \tilde{W}_{S}^{T} \\
\star & -2\left(\frac{m}{h}\right)^{2} \tilde{R}
\end{array}\right]<0
$$

where

$$
\begin{aligned}
& \tilde{\Omega}=\operatorname{sym}\left(W_{P}^{T} \tilde{W}_{S}\right)+W_{Q}^{T} \hat{Q} W_{Q}+W_{Z}^{T} \hat{Z} W_{Z} \\
& +2\left(\frac{m}{h}\right)^{2} W_{R 2}^{T}(\tilde{R}-2 \tilde{P}) W_{R 2}
\end{aligned}
$$

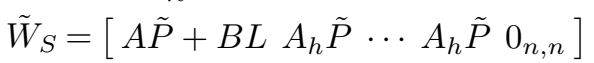

$$
\begin{aligned}
& \hat{Z}=\left[\begin{array}{cc}
\frac{h}{m} \tilde{Z} & 0_{n, n} \\
\star & -\frac{m}{h} \tilde{Z}
\end{array}\right] \\
& \hat{Q}=\left[\begin{array}{cc}
\tilde{Q} & 0_{m n, m n}, \tilde{Q} \\
\star & -\tilde{Q}
\end{array}\right], W_{R 2}=\left[\begin{array}{cc}
\frac{h}{m} I_{n}-I_{n} & 0_{n, m n}
\end{array}\right]
\end{aligned}
$$

When the above conditions are satisfied, a state feedback controller in the form of (2) is given by

$$
K=L \tilde{P}^{-1}
$$

Proof. By replacing $A$ with $A+B K$ in (7), we obtain $\operatorname{sym}\left(W_{P}^{T} P W_{S 1}\right)+W_{Q}^{T} \bar{Q} W_{Q}+W_{Z}^{T} \bar{Z} W_{Z}+W_{R 1}^{T} \bar{R} W_{R 1}<0$

where

$$
\begin{aligned}
& W_{S 1}=\left[\begin{array}{lllll}
A+B K & A_{h} \cdots A_{h} & 0_{n, n}
\end{array}\right] \\
& W_{R 1}=\left[\frac{A+B K A_{h} \cdots A_{h} 0_{n, n}}{\frac{h}{m} I_{n}-I_{n} 0_{n, m n}}\right]
\end{aligned}
$$

Using Schur complement, condition (14) is equivalent to

$$
\left[\begin{array}{cc}
\bar{\Omega} & W_{S 1}^{T} R \\
\star & -2\left(\frac{m}{h}\right)^{2} R
\end{array}\right]<0
$$

where

$$
\begin{aligned}
\bar{\Omega}= & \operatorname{sym}\left(W_{P}^{T} P W_{S 1}\right)+W_{Q}^{T} \bar{Q} W_{Q}+W_{Z}^{T} \bar{Z} W_{Z} \\
& -2\left(\frac{m}{h}\right)^{2} W_{R 2}^{T} R W_{R 2} \\
W_{R 2}= & {\left[\begin{array}{cc}
\frac{h}{m} I_{n}-I_{n} & 0_{n, m n}
\end{array}\right] }
\end{aligned}
$$

Define

$$
\begin{aligned}
& T_{1}=\operatorname{diag}\left\{P^{-1}, \cdots, P^{-1}\right\} \in \mathbb{R}^{m n \times m n} \\
& T_{2}=\operatorname{diag}\left\{T_{1}, P^{-1}, P^{-1}, R^{-1}\right\} \in \mathbb{R}^{(m+3) n \times(m+3) n}
\end{aligned}
$$

and denote

$\tilde{P}=P^{-1}, \tilde{Q}=T_{1}^{T} Q T_{1}, \tilde{Z}=\tilde{P}^{T} Z \tilde{P}, \tilde{R}=R^{-1}, L=K \tilde{P}$ Pre- and post-multiplying (15) by $T_{2}^{T}$ and $T_{2}$ derives

$$
\left[\begin{array}{cc}
\Omega_{1} & \tilde{W}_{S}^{T} \\
\star & -2\left(\frac{m}{h}\right)^{2} \tilde{R}
\end{array}\right]<0
$$

where

$$
\begin{aligned}
\Omega_{1}= & \operatorname{sym}\left(W_{P}^{T} \tilde{W}_{S}\right)+W_{Q}^{T} \tilde{Q} W_{Q}+W_{Z}^{T} \tilde{Z} W_{Z} \\
& -2\left(\frac{m}{h}\right)^{2} W_{R 2}^{T} \tilde{P}^{T} R \tilde{P} W_{R 2}
\end{aligned}
$$

Note

$$
-\tilde{P}^{T} R \tilde{P} \leq R^{-1}-2 \tilde{P}=\tilde{R}-2 \tilde{P}
$$

Then, it follows from (12) and (17) that (16) holds which guarantees the closed-loop system in (3) to be stable.

\section{ILLUSTRATIVE EXAMPLES}

In this section, some examples are provided to illustrate the applicability and reduced conservatism of the proposed approach.

Example 1: Consider a distributed delay system in (1) with following parameters:

$$
A=\left[\begin{array}{cc}
-1.2 & 0 \\
0.1 & -1.9
\end{array}\right], A_{h}=\left[\begin{array}{cc}
-1 & 0 \\
-1 & -1
\end{array}\right]
$$

The maximal allowable distributed delay $h$ satisfying (7) can be calculated by using Matlab LMI Control Toolbox. Table 1 presents a comparison, which shows that the conservatism is reduced as the number of partitions grows. Example 2: Consider a distributed delay system in (1) with following parameters:

$$
A=\left[\begin{array}{ll}
0 & 0 \\
0 & 1
\end{array}\right], A_{h}=\left[\begin{array}{cc}
-1 & -1 \\
0 & 0.9
\end{array}\right], B=\left[\begin{array}{c}
-2 \\
-1.5
\end{array}\right], h=1
$$

By computing the Laplace transform of the system in (1), we get that its characteristic polynomial is

$$
\operatorname{det}\left[s I-A-\frac{1-e^{-h s}}{s} A_{h}\right]=0
$$

By introducing parameters into above equation, we calculate one of the characteristic roots is $1.4172>0$ which is an unstable root. The state responses of the system in (1) is given in Fig. 1. which shows that it is unstable with above parameters. However, the system can be stabilized by using Theorem 2. By solving the LMI in (12) with 
Table 1. Allowable maximum distributed delay $h$ obtained by different methods

\begin{tabular}{|c|c|c|c|c|c|c|c|c|}
\hline \multirow{2}{*}{ Methods } & \multirow{2}{*}{ Li and Zhu [2008] } & \multirow{2}{*}{ Sun et al. [2009] } & \multirow{2}{*}{ Wu and Zhou [2008] } & \multicolumn{5}{|c|}{ Theorem 1} \\
\hline & & & & $m=1$ & $m=2$ & $m=3$ & $m=5$ & $m=7$ \\
\hline$h$ & 1.2000 & 2.0839 & 1.2000 & 2.0839 & 2.6969 & 3.1309 & 3.7196 & 4.0932 \\
\hline
\end{tabular}

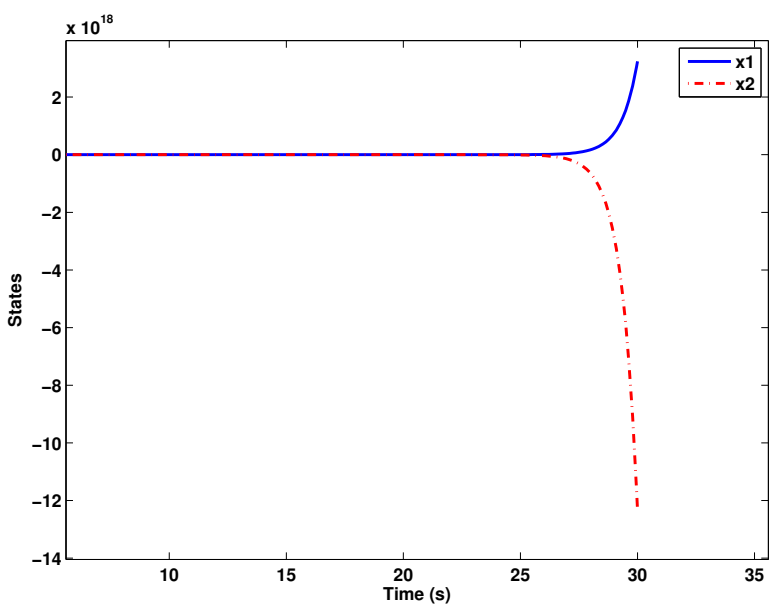

Fig. 1. State responses of open-loop system

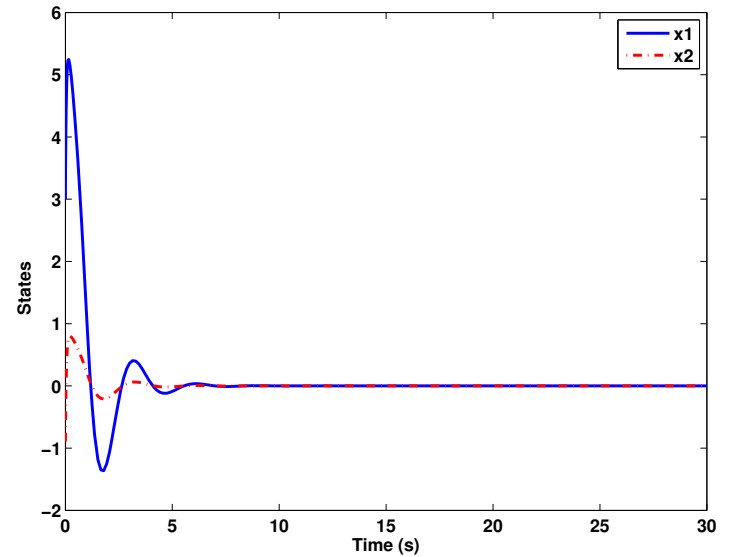

Fig. 2. State responses of closed-loop system

partitioning number $m=3$, the following matrix variables can be calculated

$$
\tilde{P}=\left[\begin{array}{cc}
43.4461 & 8.5881 \\
8.5881 & 2.9337
\end{array}\right], L=\left[\begin{array}{ll}
42.6283 & 33.6348
\end{array}\right]
$$

Then, the state feedback controller can be calculated

$$
K=L P^{-1}=[-3.050220 .3941]
$$

The state responses of closed-loop system is given in Fig. 2. which demonstrates the applicability of our controller design method. Moreover, to illustrate the reduced conservatism of our result, the feedback controllers obtained for different $m$ are given in Table 2 .

\section{CONCLUSIONS}

In this paper, the problem of delay-dependent stabilization for continuous-time systems with distributed delay has been investigated. The delay-dependent conditions in terms of LMIs have been proposed by employing the integral partitioning method. Based on this, a state-feedback controller has been designed to guarantee the closed-loop system is stable. Moreover, the results obtained in this paper will become less conservative as the partition interval become smaller. Finally, some examples are given to demonstrate the reduced conservatism and the applicability of our methods.

\section{REFERENCES}

W. H. Chen and W. X. Zheng. Delay-dependent robust stabilization for uncertain neutral systems with distributed delays. Automatica, 43:95-104, 2007.

B. Du and J. Lam. Stability analysis of static recurrent neural networks using delay-partitioning and projection. Neural Networks, 22:343-347, 2009.

L. Dugard and E. I. Verriest. Stability and Control of Time-delay Systems. Springer-Verlag, London, U.K., 1998.

Z. Fei, H. Gao, and P. Shi. New results on stabilization of Markovian jump systems with time delay. Automatica, 45:2300-2306, 2009.

Z. Feng, J. Lam, H. Gao, and B. Du. Delay-dependent robust $H_{\infty}$ controller synthesis for discrete singular delay systems. Int. J. Robust \& Nonlinear Control, Published online: 20 DEC 2010.

Y. A. Fiagbedzi and A. E. Pearson. A multistage reduction technique for feedback stabilizing distributed time-lag systems. Automatica, 23:311-326, 1987.

E. Fridman. New Lyapunov-Krasovkii functionals for stabiliy of linear retarded and neutral type systems. Systems 8 Control Letters, 43:309-319, 2001.

E. Fridman. $H_{\infty}$ control of distributed and discrete delay systems via discretized Lyapunov functional. European J. Contr., 1:1-11, 2009.

E. Fridman, U. Shaked, and K. Liu. New conditions for delay-derivative-dependent stability. Automatica, 45: 2723-2727, 2009.

H. Gao, T. Chen, and J. Lam. A new delay system approach to network based control. Automatica, 44:39$52,2008$.

F. Gouaisbaut and D. Peaucelle. Delay-dependent stability analysis of linear time delay systems. In IFAC Workshop on Time Delay System, L'Aquila, Italy, 2006.

$\mathrm{K} . \mathrm{Gu}$. An integral inequality in the stability problem of time-delay systems. In Proceedings of 39th IEEE Conference on Decision and Control, pages 2805-2810, Sydney, Australia, 2000.

K. Gu. An improved stability criterion for systems with distributed delays. Int. J. Robust \& Nonlinear Control, 13:819-831, 2003.

Y. He, Q. G. Wang, C. Lin, and M. Wu. Delay-rangedependent stability for systems with time-varying delay. Automatica, 43:371-376, 2007. 
Table 2. Feedback controllers obtained for different $m$

\begin{tabular}{|c|c|c|c|}
\hline Theorem 2 & $m=1$ & $m=2$ & $m=3$ \\
\hline$K$ & infeasible & {$\left[\begin{array}{ll}-0.8583 & 7.8517\end{array}\right]$} & {$\left[\begin{array}{ll}-3.0502 & 20.3941\end{array}\right]$} \\
\hline
\end{tabular}

X. Jiang and Q. L. Han. New stability criteria for linear systems with interval time-varying delay. Automatica, 44:2680-2685, 2008.

H. Li, B. Chen, Q. Zhou, and S. Fang. Robust exponential stability for uncertain stochastic neural networks with discrete and distributed time-varying delays. Physics Letter A, 372:3385-3394, 2008.

H. Li, B. Chen, C. Lin, and Q. Zhou. Mean square exponential stability of stochastic fuzzy hopfield neural networks with discrete and distributed time-varying delays. Neurocomputing, 72:2017-2023, 2009.

X. Li and C. E. de Souza. Delay-dependent robust stability and stabilization of uncertain linear delay system: A linear matrix ineqality approach. IEEE Trans. Automat. Control, 42:1144-1148, 1997.

$\mathrm{X}$. Li and X. Zhu. Stability analysis of neutral systems with distributed delays. Automatica, 44:2197-2201, 2008.

Y. S. Moon, P. Park, W. H. Kwon, and Y. S. Lee. Delay-dependent robust stabilization of uncertain statedelayed systems. Int. J. Control, 74:1447-1455, 2001.

H. Shao. New delay-dependent criteria for systems with interval delay. Automatica, 45:744-749, 2009.

Y. S. Suh, H. J. Kang, and Y. S. Ro. Stability condition of distributed delay systems based on an analytic solution to Lyapunov functional equations. Asian Journal of Control, 8:91-96, 2006.

J. Sun, J. Chen, G. Liu, and D. Rees. On robust stability of uncertain neutral systems with discrete and distributed delays. In Proceedings of American Control Conference, pages 5469-5473, Hyatt Regency Riverfront, St. Louis, MO, USA, 2009.

Z. Wang, Y. Liu, and X. Liu. $H_{\infty}$ filtering for uncertain stochastic time-delay systems with sector-bounded nonlinearities. Automatica, 44:1268-1277, 2008.

L. Wu, P. Shi, C. Wang, and H. Gao. Delay-dependent robust $H_{\infty}$ and $L_{2}-L_{\infty}$ filtering for LPV systems with both discrete and distributed delays. IEE Proc. Control Theory Appl., 153:483-492, 2006.

L. Wu, C. Wang, and H. Gao. Robust $H_{\infty}$ control of uncertain distributed delay systems: Parameter-dependent Lyapunov functional approach. Dynamics Continuous Discrete Impul. Systems. Ser. B: Appl. Algorithms, 14: 155-173, 2007.

Z. Wu and W. Zhou. Delay-dependent robust stabilization for uncertain singular systems with discrete and distributed delays. Journal of Control Theory and Applications, 6:171-176, 2008.

L. Xie, E. Fridman, and U. Shaked. Robust $H_{\infty}$ control of distributed delay systems with application to combustion control. IEEE Trans. Automat. Control, 46:19301935, 2001.

S. Xu and T. Chen. An LMI approach to the $H_{\infty}$ filter design for uncertain systems with distributed delays. IEEE Trans. Circuits and Systems (II), 51:195-201, 2004.

S. Xu and J. Lam. Improved delay-dependent stability criteria for time-delay systems. IEEE Trans. Automat.
Control, 50:384-387, 2005

S. Xu and J. Lam. A survey of linear matrix inequality techniques in stability analysis of delay systems. Int. J. Systems Sci., 39:1095-1113, 2008.

S. Xu, J. Lam, T. Chen, and Y. Zhou. A delay-dependent approach to robust $H_{\infty}$ filtering for uncertain distributed delay systems. IEEE Trans. Signal Processing, 53:3764-3772, 2005.

D. Yue and Q. L. Han. Robust $H_{\infty}$ filter design of uncertain descriptor systems with discrete and distributed delays. IEEE Trans. Signal Processing, 52:3200-3212, 2004

D. Yue and Q. L. Han. Delay-dependent robust $H_{\infty}$ controller design for uncertain descriptor systems with time-varying discrete and distributed delays. IEE Proc. Control Theory Appl., 152:628-638, 2005.

F. Zheng and P. M. Frank. Robust control of uncertain distributed delay systems with application to the stabilization of combustion in rocket motor chambers. Automatica, 38(2):487-497, 2002. 